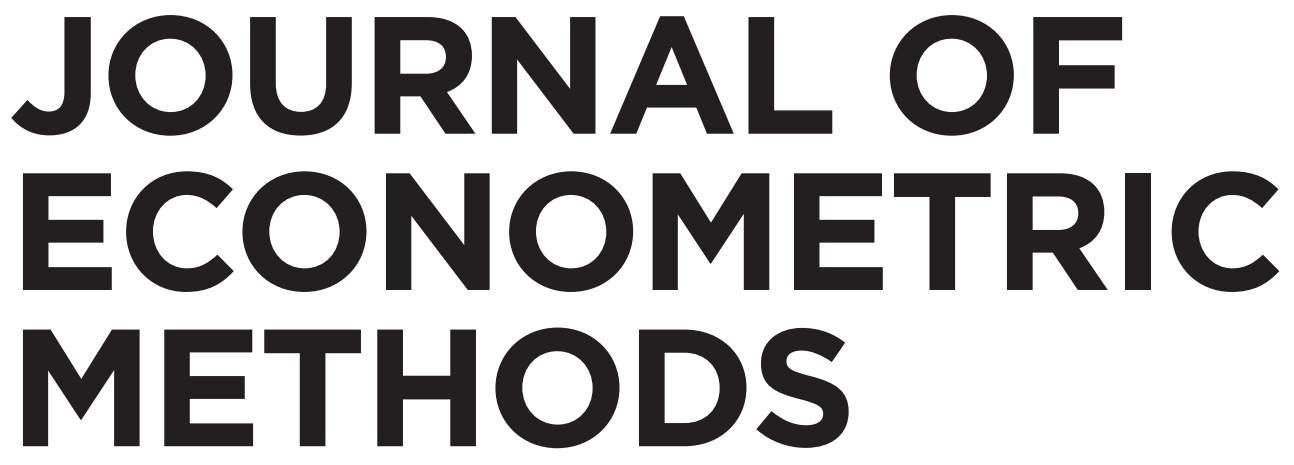

\section{EDITORS}

Raffaella Giacomini, London Tong Li, Nashville, TN
Martin Weidner, London Yoon-Jae Whang, Seoul Daniel Wilhelm, London

\title{
ASSOCIATED EDITORS
}

Stephane Bonhomme, Chicago, IL

Christian Brownlee, Barcelona

Xiaohong Chen, New Haven, CT

Sergio Firpo, São Paulo

Toru Kitagawa, London

Brendan Kline, Austin, TX

Anna Mikusheva, Cambridge, MA

Francesca Molinari, Ithaca, NY

Jose L. Montiel Olea, New York, NY

Demian Pouzo, Berkeley, CA

Zhongjun Qu, Boston, MA

Adam Rosen, Durham, NC

Yuya Sasaki, Nashville, TN

\section{DE GRUYTER}


ABSTRACTED/INDEXED IN ABDC: Australian Business Deans Council · Academic Journal Guide (CABS, UK) · Baidu Scholar · Cabells Journalytics · CNKI Scholar (China National Knowledge Infrastructure) · CNPIEC: cnpLINKer · Dimensions · EBSCO (relevant databases) · EBSCO Discovery Service · EconBiz · ECONIS · EconLit · Genamics JournalSeek · Google Scholar · IBR (International Bibliography of Reviews of Scholarly Literature in the Humanities and Social Sciences) · IBZ (International Bibliography of Periodical Literature in the Humanities and Social Sciences) · Japan Science and Technology Agency (JST) · J-Gate $\cdot$ JournalTOCs $\cdot$ KESLI-NDSL (Korean National Discovery for Science Leaders) Mathematical Reviews (MathSciNet) $\cdot$ Microsoft Academic $\cdot$ MyScienceWork $\cdot$ Naver Academic $\cdot$ Naviga (Softweco) $\cdot$ Norwegian Register for Scientific Journals, Series and Publishers · Primo Central (ExLibris) · ProQuest (relevant databases) · Publons · QOAM (Quality Open Access Market) $\cdot$ ReadCube $\cdot$ Research Papers in Economics (RePEc) $\cdot$ SCImago (SJR) - SCOPUS · Semantic Scholar · Sherpa/RoMEO $\cdot$ Summon (ProQuest) · TDNet · Ulrich's Periodicals Directory/ulrichsweb · WanFang Data · WorldCat (OCLC) · Yewno Discover · zbMATH Open

e-ISSN 2156-6674

All information regarding notes for contributors, subscriptions, Open access, back volumes and orders is available online at www.degruyter.com/jem.

RESPONSIBLE EDITORS Raffaella Giacomini, University College London, UK, e-mail: r.giacomini@ucl.ac.uk; Tong Li, Vanderbilt University, USA, e-mail: tong.li@vanderbilt.edu

PUBLISHER Walter de Gruyter GmbH, Berlin/Boston, Genthiner Straße 13, 10785 Berlin, Germany

JOURNAL MANAGER Susanne Hoeves, De Gruyter, Genthiner Straße 13, 10785 Berlin, Germany, e-mail: susanne.hoeves@degruyter.com

RESPONSIBLE FOR ADVERTISEMENTS Kevin Göthling, De Gruyter, Genthiner Straße 13, 10785 Berlin, Germany, Tel.: +49 (0)30 260 05-170, e-mail: anzeigen@degruyter.com

(C) 2021 Walter de Gruyter GmbH, Berlin/Boston, Germany

TYPESETTING TNQ Technologies, Chennai, India 